\title{
Oxidative destruction of anionite AV-17×8 using the Fenton reaction
}

\author{
M.M. Kozlova ${ }^{a^{*}}$, V.F. Markov ${ }^{a b}$, L.N. Maskaeva ${ }^{a b}$ \\ a: Ural Federal University, 620002 Mira st., 19, Ekaterinburg, Russia \\ b: Ural State Fire Service Institute of Emergency Ministry of Russia, 620022 Mira st., 22, \\ Ekaterinburg, Russia \\ * Corresponding author: marina.kozlova2014@mail.ru
}

This article belongs to the MOSM2O21 Special Issue.

(C) 2021, The Authors. This article is published in open access form under the terms and conditions of the Creative Commons Attribution (CC BY) license (http://creativecommons.org/licenses/by/4.o/)

\section{Abstract}

The kinetic studies of AV-17×8 strongly basic anionite's oxidative destruction using the Fenton reaction have been carried out. The effect of the process's temperature and the concentration of catalysts of iron(II) sulfate or copper(II) sulfate on the oxidation of anionexchange resin with hydrogen peroxide is estimated. With an increase in temperature in the range of $323-348 \mathrm{~K}$, a regular increase in the effective rate constant of oxidative anionite destruction is observed when using iron(II) sulfate by 1.5 times, and when using copper(II) sulfate - by 22 times. It was found that the obtained values of the activation energy of the anion exchanger's oxidation with the addition of copper(II) sulfate are $124.3-115.7 \mathrm{~kJ} / \mathrm{mol}$ and are characteristic of the process proceeding in the kinetic region. The nature of the change in the surface morphology of the anionite granules in the process of oxidative decomposition has been revealed.

\section{Keywords}

anion exchanger $\mathrm{AV}-17 \times 8$

hydrogen peroxide

Fenton process

process rate constant

activation energy

Received: 02.11.2021

Revised: 22.11.2021

Accepted: 23.11.2021

Available online: 26.11 .2021

\section{Introduction}

The ion exchange resins are widely used in the field of waste and wash water treatment at the nuclear power plants. As a result, the spent ion-exchange resins are formed, which are low-activity heterogeneous waste in the form of spherical granules from a cross-linked organic polymer. Over the years, the significant amounts of waste resins have accumulated at nuclear power plants, which subsequently cannot be regenerated [1]. Thus, an effective technology is needed for the disposal of the spent ionexchange resins in order to reduce their negative impact on the environment.

At present, such technologies as immobilization (cementation, bitumization, vitrification) or incineration, pyrolysis, thermal vacuum drying, and supercritical water oxidation are used to dispose of waste resins $[1,2]$. However, regular recycling technologies are characterized by significant economic costs, also there are difficulties with the transportation and storage of wastes that can be accompanied by the formation of explosive products associated with the radiolysis of organic substances and water.

An effective way of the spent ion-exchange resins removal can be oxidative destruction, which significantly reduces the concentration of organic substances. A prom- ising method is the Fenton process, based on the oxidation of organic compounds under the action of hydrogen peroxide. The catalytic additive can be ions of divalent transition metals, for example, iron(II) sulfate or copper(II) sulfate. The Fenton process is characterized by the formation of free hydroxyl radicals $\mathrm{HO}^{*}$ in the system, which have minimal selectivity to various organic substances. $\mathrm{HO}^{*}$ radicals are characterized by a high potential $(2.80 \mathrm{~V})$, and, therefore, have high oxidizing capacity [3].

The main mechanism of the classical Fenton process can be represented in the form of a redox reaction with the ferrous ions [3]:

$$
\mathrm{Fe}^{2+}+\mathrm{H}_{2} \mathrm{O}_{2} \rightarrow \mathrm{Fe}^{3+}+\mathrm{OH}^{+}+\mathrm{OH}^{-} .
$$

As a result of chain reactions and an increase in the concentration of chemically active particles, a complex organic compound RH decomposes into small fragments [3]:

$$
\begin{gathered}
\mathrm{HO}^{\bullet}+\mathrm{H}_{2} \mathrm{O}_{2} \rightarrow \mathrm{HOO}^{\bullet}+\mathrm{H}_{2} \mathrm{O}, \\
\mathrm{Fe}^{3+}+\mathrm{HOO}^{\bullet} \rightarrow \mathrm{Fe}^{2+}+\mathrm{H}^{+}+\mathrm{O}_{2}, \\
\mathrm{Fe}^{2+}+\mathrm{HOO}^{-} \rightarrow \mathrm{Fe}^{3+}+\mathrm{HOO}^{-}, \\
\mathrm{Fe}^{2+}+\mathrm{HO} \cdot \rightarrow \mathrm{Fe}^{3+}+\mathrm{OH}^{-}, \\
\mathrm{HO}^{\bullet}+\mathrm{RH} \rightarrow \mathrm{R}^{\cdot}+\mathrm{H}_{2} \mathrm{O} .
\end{gathered}
$$


The Fenton process is characterized by a high reactivity, a deep oxidation state, and sufficiently mild operating conditions. The reaction efficiency is influenced by such factors as temperature, $\mathrm{pH}$ of the medium, concentration of hydrogen peroxide and catalyst.

The oxidation of sulfonic acid cation exchangers with hydrogen peroxide, as well as strongly basic anion exchangers with tertiary trimethylammonium groups in the presence of catalytic additions of transition metal salts, was described in [4-14]. Thus, in the study [4], the complete decomposition of domestic brand anionite AV-17 $\times 8$ was achieved by the action of a 30\% solution of hydrogen peroxide with the addition of an iron(II) salt in the temperature range $363-373 \mathrm{~K}$. The works of the authors [5-14] are aimed at choosing the optimal conditions for the oxidative destruction of ion exchange resins of some foreign brands. The research [9] is devoted to the complete decomposition of a foreign brand of anion-exchange resin Amberlite INR78 by exposure to a 30\% hydrogen peroxide solution with the addition of a copper (II) salt at a temperature of $368 \mathrm{~K}$. In the above mentioned works the individual aspects of the influence such as the concentration of the oxidizing agent, the temperature of the solution, and the concentration of catalytic additives on the Fenton process were studied.

However, there is currently no information on kinetics of the ion-exchange resins oxidative destruction using the Fenton process. Previously, the studies were carried out on the oxidation of hydrogen peroxide using the Fenton reaction of the highly acidic universal cation exchanger KU-2-8 [15]. This works object is to the study of the kinetics of catalytic oxidative destruction of the domestic anionite $\mathrm{AV}-17 \times 8$ using the Fenton process.

\section{Experimental}

The object of the study was the strongly basic anion exchanger AV-17×8 (GOST 20301-74), the crosslinked copolymer of styrene and divinylbenzene. The diameters of spherical resin granules are in range of $315-1250 \mu \mathrm{m}$, the content of the working fraction is not less than $95 \%$, the uniformity coefficient is 1.6 , and the specific volume is $3.0 \pm 0.3 \mathrm{~cm}^{3} / \mathrm{g}$. For the oxidation of the AV $-17 \times 8$ anionite, we used an environmentally friendly oxidizing agent hydrogen peroxide $\mathrm{H}_{2} \mathrm{O}_{2}$, the concentration of which was determined by permanganatometry [16]. The preparation of $0.1 \mathrm{M}$ catalyst solutions was carried out using salts of iron(II) sulfate $\mathrm{FeSO}_{4} \cdot 7 \mathrm{H}_{2} \mathrm{O}$ and copper(II) sulfate $\mathrm{CuSO}_{4} \cdot 5 \mathrm{H}_{2} \mathrm{O}$.

In all experiments, the amount of anion-exchange resin was constant and was $0.5 \mathrm{~g}$ based on the weight of the airdry mass. To study the catalytic oxidation, a weighed portion of the $\mathrm{AV}-17 \times 8$ anionite was introduced into the reactors, then $10 \mathrm{ml}$ of a hydrogen peroxide solution with an $\mathrm{H}_{2} \mathrm{O}_{2}$ concentration of $20 \mathrm{vol} . \%$, then $0.001-0.005 \mathrm{~mol} / \mathrm{L}$ $\mathrm{FeSO}_{4}$ or $\mathrm{CuSO}_{4}$ was added.
The reactors were placed in the thermostat of the brand LOIP LT-105a. The process was carried out in the temperature range of 323-348 K. Each reactor was removed from the thermostat after a certain time. The remaining AV $-17 \times 8$ anionite in the reactors was thoroughly washed, filtered, dried in air for a week at room conditions, then heated in a PM-1.0-7 electric furnace for 2.5 h at the temperature of $377 \pm 1 \mathrm{~K}$. After that, it was weighed on an analytical balance VIBRA HTR-220CE with a readability of $\pm 0.0001 \mathrm{~g}$.

The study of the surface morphology of the granules of the anionite $\mathrm{AV}-17 \times 8$ was carried out by scanning electron microscopy using a JEOL JSM-6390 LA microscope.

\section{Results and discussion}

To explain the physicochemical process of $\mathrm{AV}-17 \times 8$ anionite oxidation, it is necessary to consider the patterns of the process in time, depending on the mechanism of the chemical reaction and on thermodynamic factors - the temperature and concentration of the catalyst. Let us consider the effect of temperature as a parameter that has the most significant impact on the rate of anion exchanger oxidative destruction.

The kinetic studies of the AV-17 $\times 8$ anionite catalytic oxidation with hydrogen peroxide were carried out with the addition of $0.001-0.005 \mathrm{~mol} / \mathrm{L}$ iron(II) sulfate and copper(II) sulfate. The dependences of the relative weight loss of the anion exchange resin on the exposure time of $20 \mathrm{vol} \%$ hydrogen peroxide with the addition of $0.002 \mathrm{~mol} / \mathrm{L} \mathrm{FeSO}_{4}$ at temperatures from 323 to $343 \mathrm{~K}$ are shown in Fig. 1a. It is observed that the process of the anionite destruction by hydrogen peroxide with iron(II) sulfate proceeds relatively slowly, and with a decrease in the reaction temperature, the longer induction period occurs. So, at $343 \mathrm{~K}$ in $360 \mathrm{~min}$, only $36 \%$ of the anion exchanger was dissolved. The lowering of the temperature to $333 \mathrm{~K}$ leads to the decomposition of $17 \%$ of resin within $210 \mathrm{~min}$. At $323 \mathrm{~K}$, $10 \%$ of the anion exchanger is oxidized in $270 \mathrm{~min}$.

With the use of copper(II) sulfate as a catalytic additive at the temperatures in range of $323-348 \mathrm{~K}$, the complete oxidation of the $A V-17 \times 8$ anionite was achieved (Fig. 1b). The graph shows how the duration of the induction period decreases with increasing temperature. At $348 \mathrm{~K}$ the intense oxidation of the anion exchange resin is observed during the first $24 \mathrm{~min}$. The decrease in the working temperature of the solution to 343 and $333 \mathrm{~K}$ leads to the complete decomposition of the resin after 35 and $110 \mathrm{~min}$, respectively. At $323 \mathrm{~K}$ after $270 \mathrm{~min}$, the resin mass loss was $\sim 92 \%$.

Thus, an increase in the process temperature from $323 \mathrm{~K}$ to $348 \mathrm{~K}$ significantly influences the rate of anion exchange resin decomposition. It should be noted that increasing the catalyst concentration that is iron(II) sulfate or copper(II) sulfate at a given temperature does not significantly accelerate the decomposition of the $\mathrm{AV}-17 \times 8$ anionite. 

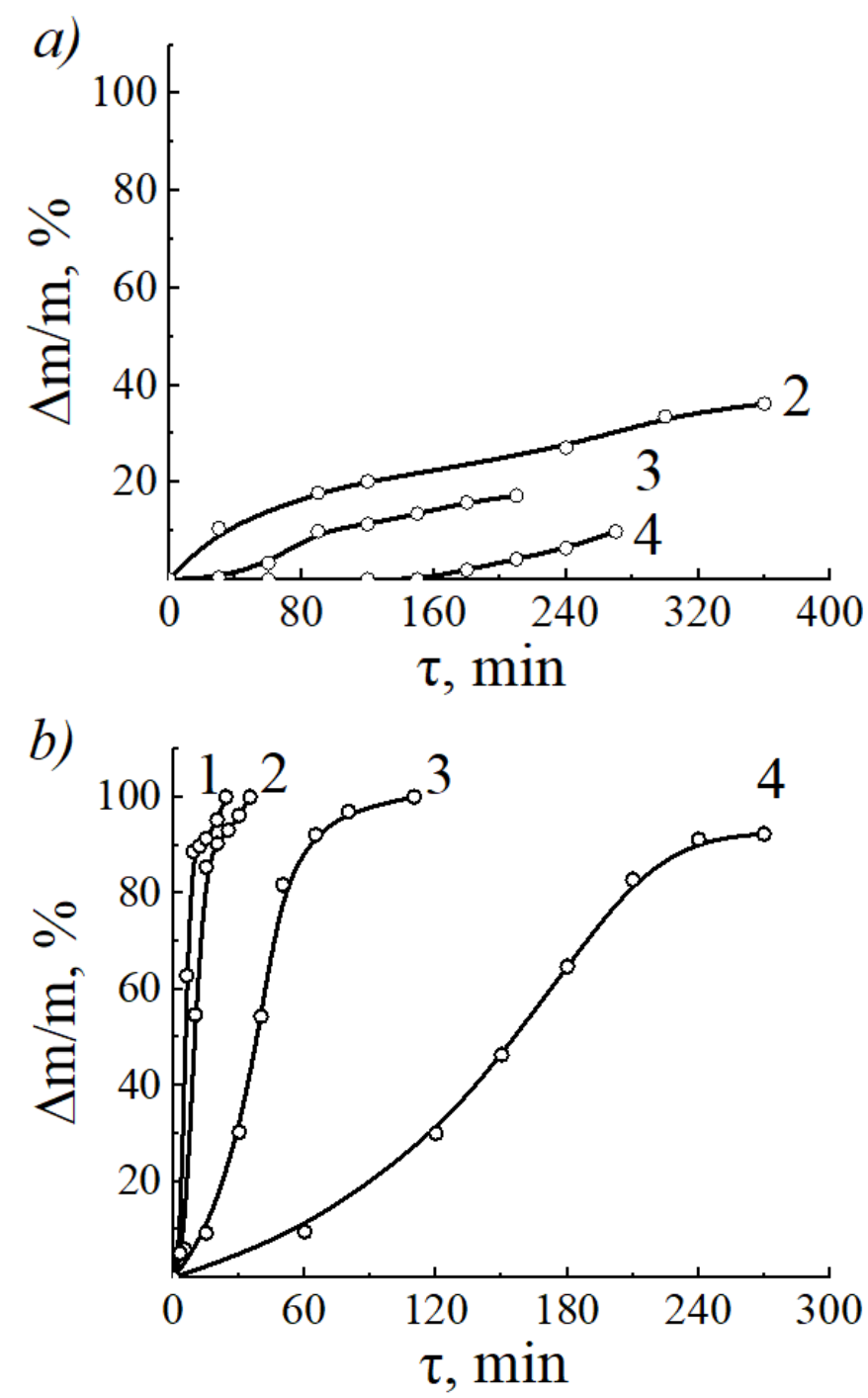

Fig. 1 Kinetic curves of the AV-17 $\times 8$ anionite relative weight loss in $20 \% \mathrm{H}_{2} \mathrm{O}_{2}$ with the addition of $0.002 \mathrm{~mol} / \mathrm{L} \mathrm{FeSO}_{4}$ (a) and $0.003 \mathrm{CuSO}_{4} \mathrm{~mol} / \mathrm{L}(\mathrm{b})$ at a temperature, $\mathrm{K}: 348$ (1), 343 (2), 333 (3), $323(4)$

The effect of catalytic additions of copper and iron salts, according to studies $[6-9,11,14]$, is based on an increase in the concentration of active oxygen during the decomposition of hydrogen peroxide, which promotes more active resin oxidative destruction. In the works $[6,9]$, the activities of catalytic additives in the $\mathrm{Fe}^{2+} / \mathrm{H}_{2} \mathrm{O}_{2}$ and $\mathrm{Cu}^{2+} / \mathrm{H}_{2} \mathrm{O}_{2}$ solutions have been compared and it is concluded that copper(II) ions have a stronger catalytic effect in the process of the anion exchanger oxidation compared to iron(II) ions.
To determine the rate of the heterogeneous process in the "anionite-solution" system, it is necessary to take into account the interfacial area that changes during oxidative destruction, as well as the geometry of the spherical particles of the anion-exchange resin. The reaction rate, that is, the loss of anionite's mass, can be determined according to the equation:

$$
-\frac{d m}{d \tau}=k F C
$$

where $m$ is the mass of the anionite $\mathrm{AV}-17 \times 8$ granule at the time $\tau, F$ is its surface area, $C$ is the concentration of $\mathrm{H}_{2} \mathrm{O}_{2}$, $k$ is the rate constant of the oxidation reaction.

By transforming Eq. (7), it is possible to obtain the dependence of the change in the mass of the anion resin granule on the duration of the process of its oxidation " $m^{1 / 3}$ - $\tau$ " [17]. The processing of the experimental results, carried out in coordinates " $m^{1 / 3}-\tau$ ", allows taking into account the heterogeneous nature of the oxidation reaction and the spherical shape of the anionite $A V-17 \times 8$ granules.

The effective rate constant of the process $k$ was determined from the slope of the obtained straight line in the coordinates " $m^{1 / 3}-\tau$ ". Table 1 shows the calculated values of the effective rate constants of the anion exchanger oxidative destruction process depending on the temperature and the concentration of catalytic additives. From the calculated values with the addition of 0.002 $\mathrm{mol} / \mathrm{L} \mathrm{FeSO}_{4}$ catalyst, it shows that an increase in the process temperature from 323 to $343 \mathrm{~K}$ leads to an increase in the rate constant by a factor of 1.5 . With an increase in the content of the catalytic additive from 0.001 to $0.005 \mathrm{~mol} / \mathrm{L}$, the rate constant of the process increases by a factor of 1.2.

According to the obtained values of the process effective rate constant with the addition of $0.002 \mathrm{~mol} / \mathrm{L} \mathrm{CuSO}_{4}$, and with increase in the process temperature from 323 to $348 \mathrm{~K}$ the rate constant is increasing by a factor of 23 . At $0.003,0.004$, and $0.005 \mathrm{~mol} / \mathrm{L} \mathrm{CuSO}_{4}$, the effective rate constant of the process increases by a factor of 22 . With a decrease in the concentration of the catalytic additive to $0.001 \mathrm{~mol} / \mathrm{L} \mathrm{CuSO}_{4}$, an increase in the rate constant by a factor of 18 is observed. An increase in the concentration of the $\mathrm{CuSO}_{4}$ catalyst from 0.001 to $0.005 \mathrm{~mol} / \mathrm{L}$ slows down the increase in the process rate constant, increasing it by no more than 1.3-2.2 times.

Table 1 Effective process rate constant $\left(k \times 10^{3}, \mathrm{~g}^{1 / 3} \mathrm{~min}^{-1}\right)$ oxidative destruction of the anionite AV-17 $\times 8$, depending on the temperature and concentration of catalytic additives when using $20 \mathrm{vol} . \%$ hydrogen peroxide

\begin{tabular}{|c|c|c|c|c|c|c|c|c|c|c|}
\hline \multirow{2}{*}{$T, \mathrm{~K}$} & \multicolumn{5}{|c|}{$\left[\mathrm{FeSO}_{4}\right], \mathrm{mol} / \mathrm{L}$} & \multicolumn{5}{|c|}{$\left[\mathrm{CuSO}_{4}\right], \mathrm{mol} / \mathrm{L}$} \\
\hline & 0.001 & 0.002 & 0.003 & 0.004 & 0.005 & 0.001 & 0.002 & 0.003 & 0.004 & 0.005 \\
\hline 323 & - & 0.23 & 0.25 & 0.34 & 0.35 & 2.15 & 2.71 & 2.93 & 3.20 & 3.25 \\
\hline 333 & 0.20 & 0.24 & 0.25 & - & - & 5.82 & 7.88 & 9.13 & 10.29 & 12.67 \\
\hline 343 & 0.27 & 0.34 & - & - & - & 32.07 & 33.49 & 35.99 & 42.13 & 41.83 \\
\hline 348 & - & - & - & - & - & 38.41 & 63.73 & 65.96 & 70.67 & 72.75 \\
\hline
\end{tabular}


Using the calculated values of the effective rate constants, the activation energy $E_{a}$ of the process of the anionite oxidative destruction was determined by the graphical solution of the Arrhenius equation in the coordinates " $\ln k-10^{3} / T^{\prime \prime}$. According to the data presented in Table 2, the activation energies of the anionite oxidation process with the addition of the $\mathrm{CuSO}_{4}$ catalyst are in the range from 124.3 to $115.7 \mathrm{~kJ} / \mathrm{mol}$, which indicates that the process is of the kinetic type.

Table 2 Activation energy of the process of oxidative destruction of the AV- $17 \times 8$ anionite in $20 \%$ hydrogen peroxide at various concentrations of the $\mathrm{CuSO}_{4}$ catalyst

\begin{tabular}{lccccc}
\hline $\begin{array}{l}{[\mathrm{CuSO}} \\
\mathrm{mol} / \mathrm{L}\end{array}$ & 0.001 & 0.002 & 0.003 & 0.004 & 0.005 \\
\hline$E_{a}, \mathrm{~kJ} / \mathrm{mol}$ & 124.3 & 118.1 & 116.4 & 115.7 & 116.2 \\
\hline
\end{tabular}

The surface of the anionite $\mathrm{AV}-17 \times 8$ granules in the process of catalytic oxidative destruction has been investigated. For comparison, Fig. 2a shows a relatively smooth and practically undeformed surface of granules before oxidation. Fig. 2b shows an electron microscopic image of a surface of granules after $2.5 \mathrm{~h}$ of contact at the temperature of $343 \mathrm{~K}$ with a 20 vol.\% aqueous solution of hydrogen peroxide containing $0.002 \mathrm{~mol} / \mathrm{L} \mathrm{FeSO}_{4}$, which corresponds to a loss of $20 \%$ of granule's mass. Fig. $2 \mathrm{c}$ shows a surface of anionite granules after exposure to a 20 vol.\% aqueous solution of $\mathrm{H}_{2} \mathrm{O}_{2}$ containing $0.005 \mathrm{~mol} / \mathrm{L} \mathrm{CuSO}_{4}$. As a result of contact for $10 \mathrm{~min}$ at the temperature of $343 \mathrm{~K}$, the weight loss of the anionite was $85 \%$. In Fig. 2(b, c), the local changes can be observed on the resin surface. At the same time, the sorbent granule changed its shape, volume, and its surface was covered with cracks, which may indicate the destruction of the crosslinks of the $\mathrm{AV}-17 \times 8$ anionite in the process of oxidative destruction and a decrease in its mechanical strength.

\section{Conclusions}

Thus, the kinetic studies of the AV-17 $\times 8$ anionite catalytic oxidative destruction by hydrogen peroxide showed that the introduction of $0.001-0.005 \mathrm{~mol} / \mathrm{L}$ of the copper(II) sulfate catalyst significantly increases the intensity of the oxidation process as compared to the addition of $0.001-0.005 \mathrm{~mol} / \mathrm{L}$ of iron(II) sulfate. It was found that in the presence of $0.002 \mathrm{~mol} / \mathrm{L}$ catalytic additives in a $20 \%$ aqueous solution of hydrogen peroxide in the temperature range $323-348 \mathrm{~K}$ the rate constant of the oxidative decomposition of the anion exchanger increases by a factor of 1.5 when using iron(II) sulfate at an operating temperature of $343 \mathrm{~K}$, and with the introduction of copper(II) sulfate at $348 \mathrm{~K}$ - by a factor of 23 . The calculated activation energies of the process of oxidative destruction of the AV $-17 \times 8$ anionite by hydrogen peroxide with the addition of copper(II) sulfate are in the range $124 \cdot 3-115.7 \mathrm{~kJ} / \mathrm{mol}$, which is typical for the kinetic type process.
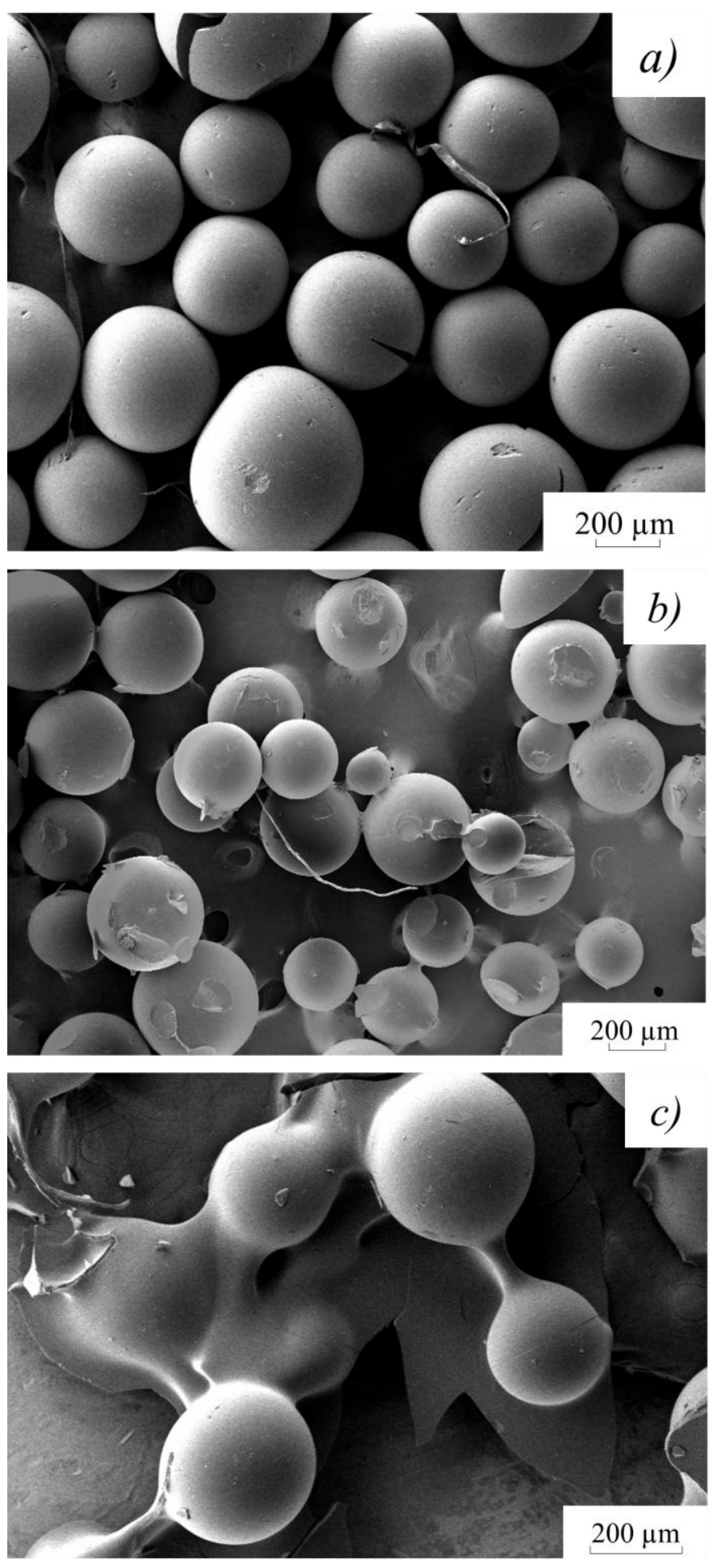

Fig. 2 Electron microscopic images of the surface of the anionite AV-17 $\times 8$ before oxidation (a), after exposure to $20 \% \mathrm{H}_{2} \mathrm{O}_{2}$ with the addition of $\mathrm{FeSO}_{4}$ (b) and with the addition of $\mathrm{CuSO}_{4}$ (c)

\section{References}

1. Smolnikov MI, Markov VF, Maskaeva LN, Bobylev AE, Mokrousova OA. Utilization problems of spent ion-exchange resins of nuclear power plants. Butlerov Commun. 2017;49(3):119-134. doi:jbc-01/17-49-3-119

2. WangJ, Wan $Z$. Treatment and disposal of spent radioactive ion-exchange resins produced in the nuclear industry. Prog Nucl Energy. 2015;78:47-55. doi:10.1016/i.pnucene.2014.08.003

3. Babuponnusami A., Muthukumar K. A review on Fenton and improvements to the Fenton process for wastewater treat- 
ment. J Environ Chem Eng. 2014;2(1):557-572.

doi: $10.1016 /$ j.jece.2013.10.011

4. Kuznetsov AE, Knyazev OV, Maraev IY, Manakov MN. Biotechnological destruction of ion exchange resins. Biotechnol. 2000;16(1):66-77.

5. Zahorodna M, Bogoczek R, Oliveros E, Braun AM. Application of the Fenton process to the dissolution and mineralization of ion exchange resins. Catal Today. 2007;129(1-2):200-206. doi:10.1016/i.cattod.2007.08.014

6. Gunale TL, Mahajani VV, Wattal PK, Srinivas C. Liquid phase mineralization of gel-type anion exchange resin by a hybrid process of Fenton dissolution followed by sonication and wet air oxidation. Asia-Pacific J Chem Eng. 2009;4(1):90-98. doi:10.1002/apj.214

7. Gunale TL, Mahajani VV, Wattal PK, Srinivas C. Studies in liquid phase mineralization of cation exchange resin by a hybrid process of Fenton dissolution followed by wet oxidation. Chem Eng J. 2009;148(2-3):371-377.

doi:10.1016/i.cej.2008.09.018

8. Wan Z, Xu L, Wang J. Disintegration and dissolution of spent radioactive cationic exchange resins using Fenton-like oxidation process. Nucl Eng Des. 2015;291:101-108. doi:10.1016/i.nucengdes.2015.05.009

9. Wan Z, Xu L, Wang J. Treatment of spent radioactive anionic exchange resins using Fenton-like oxidation process. Chem Eng J. 2016;284:733-740. doi:10.1016/j.cej.2015.09.004

10. de Araujo LG, Marumo JT. Reaction of ion exchange resins with fenton's reagent. Environments - MDPI. 2018;5(11):110. doi:10.3390/environments5110123
11. Xu L, Meng X, Li M, Li W, Sui Z, Wang J, Yang J. Dissolution and degradation of nuclear grade cationic exchange resin by Fenton oxidation combining experimental results and DFT calculations. Chem Eng J. 2019;361:1511-1523. doi:10.1016/i.cej.2018.09.169

12. Huang CP, Tsai MT, Li YJ, Huang YH, Chung TY. Oxidative dissolution of cation ion exchange resin by the Fenton process using a fluidized bed reactor. Prog Nucl Energy. 2020;125:1-8. doi:10.1016/i.pnucene.2020.103377

13. Feng W, Li J, An H, Wang Y. Degradation of spent radioactive ion exchange resins and its mechanisms by fenton process. J Renew Mater. 2020;8(10):1283-1293.

doi:10.32604/jrm.2020.011000

14. Hafeez MA, Jeon J, Hong S, Hyatt N, Heo J, Um W. Fenton-like treatment for reduction of simulated carbon-14 spent resin.J Environ Chem Eng. 2021;9(1):1-9.

doi:10.1016/i.jece.2020.104740

15. Kozlova MM, Markov VF, Maskaeva LN, Smol'nikov MI, Savinykh SD. Kinetics of the Oxidative Degradation of KU-2 $\times 8$ Cation-Exchange Resin Using Hydrogen Peroxide. Russ J Phys Chem A. 2020;94(12):2450-2458. doi:10.1134/So036024420120146

16. Pilipenko AT, Pyatnitsky IV. Analiticheskaya khimiya [Analytical chemistry]. Moscow: Chemistry, 1990.480 p. Russian.

17. Dyachenko AN, Shagalov VV. Khimicheskaya kinetika geterogennykh protsessov [Chemical kinetics heterogeneous processes]. Tomsk: Tomsk Poly Technical University, 2014. 102 p. Russian. 